\title{
A Two-Echelon Green Supply Chain with Simultaneous Pickup and Delivery
}

\author{
Setareh Abedinzadeh ${ }^{1, ~ *}$, Ali Ghoroghi ${ }^{2}$, Sara Afshar ${ }^{3}$, Mahsa Barkhordari ${ }^{3}$ \\ ${ }^{1}$ Department of Industrial Engineering, University of Science and Culture, Tehran, Iran \\ ${ }^{2}$ Department of Computer Engineering, University of Science and Culture, Tehran, Iran \\ ${ }^{3}$ Department of Industrial Engineering, Science and Research Branch, Islamic Azad University, Tehran, Iran \\ Email address: \\ abedinzadeh.setareh@gmail.com (S. Abedinzadeh) \\ ${ }^{*}$ Corresponding author
}

\section{To cite this article:}

Setareh Abedinzadeh, Ali Ghoroghi, Sara Afshar, Mahsa Barkhordari. A Two-Echelon Green Supply Chain with Simultaneous Pickup and Delivery. International Journal of Transportation Engineering and Technology. Vol. 3, No. 2, 2017, pp. 12-18.

doi: $10.11648 /$ j.jijtet.20170302.11

Received: April 27, 2017; Accepted: June 13, 2017; Published: July 14, 2017

\begin{abstract}
Supply chain network determines the structure of a chain and affects its costs and performance. It deals with a variety of decisions such as determining number, size and location of facilities in a supply chain (SC) as well as fulfilling customers demand. In this paper, we considered a variant of the Location-Routing Problem (LRP) with consideration of green aspects, namely the green LRP with simultaneous pickup and delivery (GLRPSPD). This specific problem seeks to minimize total cost by simultaneously locating the distribution centers and designing the vehicle routes that satisfy pickup and delivery demand of each customer at the same time, in a way that ecological aspects are observed. The problem was formulated as a mixed integer programming (MIP) model, which could then be solved using general algebraic modelling system (GAMS) optimization software to determine the best vehicle routs and the optimal number of utilized vehicles.
\end{abstract}

Keywords: Location-Routing Problem, Green Routing, Simultaneous Pickup, Delivery, GAMS Optimization Software

\section{Introduction}

Supply chain network determines the structure of a chain and affects its costs and performance. An important aspect of designing a distribution network is the determination of the locations of facilities such as warehouses, depots, distribution centers, etc. Classical facility location models assume that each customer (i.e. customer, market, etc.) is served on a straight-and-back basis on a given route while computing distribution cost. The location-routing problem (LRP) deals with determining the location of facilities and the routes of the vehicles for serving the customers under some constraints such as facility and vehicle capacities, route length, etc. to satisfy demands of all customers and to minimize the total cost including routing costs, vehicle fixed costs, facility fixed costs and facility operating costs. In its general form, the LRP assumes that customers have only delivery demand and it is interested in how to distribute the goods to customers with a fleet of vehicles, which are stationed in the opened depots. However, in practice, customers can have pickup and delivery demands and they often request that both demands should be met at the same time. By taking into consideration this kind of demand structure of customers, a variant of the LRP called the LRP with simultaneous pickup and delivery (LRPSPD) is applied in 2012 which was introduced by Karaoglan et al [4].

As mentioned above, before 2012 there was no previous study on LRPSPD in literature. In this paper, we considered a variant of the Location-Routing Problem (LRP) with consideration of green aspects, namely the green LRP with simultaneous pickup and delivery (GLRPSPD). This specific problem seeks to minimize total cost by simultaneously locating the distribution centers and designing the vehicle routes that satisfy pickup and delivery demand of each customer at the same time, in a way that ecological aspects are observed. To the best of our knowledge, this paper is the first research that considers GLRPSPD. GLRPSPD is a combination of facility location problem (FLP), vehicle routing problem with simultaneous pickup and delivery (VRPSPD) and green routing. Since the problem is unknown 
in literature, we explain the literature of FLP, VRPSPD and green routing.

We refer the interested readers to the review papers of Melo et al and Smith et al for FLP [6], [10]. The followings are the related literature of VRPSPD. Min et al first recognized the possibility of simultaneous deliveries and pickups at the same node. The main objective of the paper was to develop a model and a solution procedure efficient enough to handle real-world variants. They described and conducted a case study dealing with a public library distribution system in Franklin County, Ohio. The final result of the case study indicated that substantial time/distance savings can be achieved by using the proposed model and solution procedure [7]. The problem of simultaneously allocating customers to depots, finding the delivery routes and determining the vehicle fleet composition was addressed by Salhi et al. A multi-level composite heuristic was proposed and two reduction tests are designed to enhance its efficiency [9]. Zachariadis et al presented a meta-heuristic methodology for the Capacitated Vehicle Routing Problem with two-dimensional loading constraints (2L-CVRP). 2LCVRP is a generalisation of the Capacitated Vehicle Routing Problem, in which customer demand is formed by a set of two-dimensional, rectangular, weighted items. The purpose of the problem was to produce the minimum cost routes, starting and terminating at a central depot, to satisfy the customer demand. Furthermore, the transported items must be feasibly packed into the loading surfaces of the vehicles. They proposed a meta-heuristic algorithm which incorporates the rationale of Tabu Search and Guided Local Search [16]. Subramanian et al presented a parallel approach for solving the Vehicle Routing Problem with Simultaneous Pickup and Delivery (VRPSPD). The parallel algorithm was embedded with a multi-start heuristic which consists of a variable neighbourhood descent procedure, with a random neighbourhood ordering (RVND), integrated in an iterated local search (ILS) framework. The experiments were performed in a cluster with a multi-core architecture using up to 256 cores. The results obtained on the benchmark problems show that the proposed algorithm not only improved several of the known solutions, but also presented a very satisfying scalability [11]. Tasan et al presented a vehicle routing problem with simultaneous pick-up and deliveries, which considers simultaneous distribution and collection of goods to/from customers, is an extension of the capacitated vehicle routing problem. They proposed a genetic algorithm based approach to this problem. Computational example was presented with parameter settings in order to illustrate the proposed approach. Moreover, performance of the proposed approach was evaluated by solving several test problems [12].

The rest of literature of this part is applicable to green routing. Christie et al focused on the estimation of emission reduction benefits and the potential energy savings that can be realized with logistics optimization, particularly those resulting from computerized vehicle routing and scheduling (VRS) of pickups and deliveries at a trucking terminal. The objective of the problem was to quantify the benefits and potential efficiency gains in terms of emission reduction with computerized VRS optimization (CVRSO) implementation by making a comparative analysis between different CVRSO methods and existing manual VRS methods. After setting up the CVRSO system, different optimization criteria were used and it was found that, in addition to significant cost savings, a significant benefit in terms of a reduction in energy use and corresponding pollution reduction is achieved by minimizing operational costs (by combining fixed costs and both distance-related and time-related costs, and optimizing the routing and scheduling for the fleet of vehicles) [1]. Yong et al considered the designing the optimal set of routes for fleet of vehicles in order to serve a given set of customers. They considered vehicle managers in the vehicle routing schedules which minimize fuel consumption. This paper provided a new vehicle routing problem based on reducing fuel consumption. That is, the optimization objective of the vehicle routing problem which is to minimize the fuel consumption. The paper also gave the corresponding vehicle routing problem, the optimization objective of which is to minimize the travel distance. Through a numerical example, the two problems were compared. The result showed that a different vehicle routing schedule is probable found if the optimization objective was to minimize fuel consumption other than to minimize travel distance [15]. Figliozzi et al studied approximations to the average length of vehicle routing problems (VRP) with time window, route duration, and capacity constraints. Using asymptotic properties of vehicle routing problems and the average probability of successfully sequencing a customer with time windows a new expression to estimate VRP distances was developed. The increase in the number of routes when time constraints are added was modeled probabilistically. This paper introduced the concept of the average probability of successfully sequencing a customer with time windows. The approximation proposed was tested in instances with different customer spatial distributions, depot locations and number of customers. Regression results indicated that the proposed approximation was not only intuitive but also predicts the average length of VRP problems with a high level of accuracy [2]. Urquhart et al used an evolutionary Multi-Objective Algorithm (MOA) investigate the trade-off between $\mathrm{CO} 2$ savings, distance and number of vehicles used in a typical vehicle routing problem with Time Windows (VRPTW). A problem set was derived containing three problems based on accurate geographical data which encapsulates the topology of streets as well as layouts and characteristics of junctions. Results obtained using a wellknown MOA with twin objectives show that it was possible to save up to $10 \% \mathrm{CO} 2$, depending on the problem instance and ranking criterion used [13]. Ilgin et al presented an important review of the development of research in Environmentally Conscious Manufacturing and Product Recovery (ECMPRO) and provided a state of the art survey of published work [3]. Kuo et al started to realize the importance of environmental protection, and in particular 
the problem of global warming. Consequently, many governments started to view decreasing carbon emissions as a priority. Green transportation was one of the policies that is relevant to these efforts. This paper aimed to optimize the routing plan with minimizing fuel consumption. Then a simple Tabu Search was used to optimize the routing plan and an experimental evaluation of the proposed method was performed [5]. Xiao et al added the Fuel Consumption Rate (FCR), a factor considered as a load dependant function, to the classical capacitated vehicle routing problem (CVRP) to extend traditional studies on CVRP with the objective of minimizing fuel consumption. They presented a mathematical optimization model to formally characterize the FCR considered CVRP (FCVRP) as well as a string based version for calculation. A simulated annealing (SA) algorithm with a hybrid exchange rule was developed to solve FCVRP and shows good performance on both the traditional CVRP and the FCVRP in substantial computation experiments [14]. Pradenas et al evaluated the decrease in the emission of greenhouse gases using the vehicle routing problem with backhauls and time windows by considering the energy required for each route and estimating the load and distance between customers. Using a Scatter Search (SS), problems from the literature with up to 100 randomly distributed customers were analyzed. The results indicated that the distance traveled and the transportation costs increase as the required energy decreases, but the amount of fuel consumed also decreases; therefore, the emission of greenhouse gases also decreases [8].

This paper is organized as follows. Section 2 explains the definition of the problem; in section 3 , mathematical model is developed; methodology and numerical experiments are included in section 4. Finally, the conclusions and proposals for future research are presented in section 5 .

\section{Problem Definition}

In this section, we explain the problem definition and basic assumptions to better understand the problem. We regarded a two-echelon (distribution and customer) single-product supply chain. As mentioned above, the main problem is LRPSPD based on Karaoglan et al [4]. LRPSPD can be defined as follows: let $G=(\mathrm{N}, \mathrm{A})$ be a complete directed network where $\mathrm{N}=\mathrm{N}_{0} \cup \mathrm{N}_{\mathrm{C}}$ is a set of nodes in which $\mathrm{N}_{\mathrm{O}}$ and $\mathrm{N}_{\mathrm{C}}$ represent the potential depot nodes and customers,

Sets and indices :

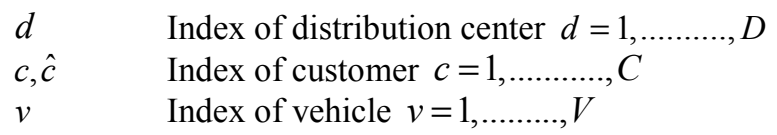

Parameters :

$\begin{array}{ll}\operatorname{cap}_{d}^{\text {dist }} & \text { Capacity of distribution center } d \\ \operatorname{cap}_{v}^{\text {veh }} & \text { Capacity of vehicle } v \\ \operatorname{dis}_{\tilde{c} c}^{c u s} & \text { Distance between customer } c \text { and customer } \hat{c}\end{array}$

respectively, and $A=\{(i, j): i, j \in N\}$ is the set of arcs. Each $\operatorname{arc}(i, j) \in \mathrm{N}$ has a nonnegative cost (distance), $\mathrm{C}_{\mathrm{ij}}$, and triangular inequality holds (i.e., $\mathrm{C}_{\mathrm{ij}}+\mathrm{C}_{\mathrm{jk}} \geq \mathrm{C}_{\mathrm{ik}}$ ). A capacity $\mathrm{CD}_{\mathrm{k}}$ and a fixed cost $\mathrm{FD}_{\mathrm{k}}$ are associated with each potential depot $\mathrm{k} \in \mathrm{N}_{\mathrm{O}}$. .An unlimited fleet of homogeneous vehicles with capacity $C V$ and fixed operating cost $F V$ including the cost of acquiring the vehicles used in the routing is available to serve the customers. It should be noted that we consider the assumption of an unlimited number of homogeneous vehicles with known capacities in order to dimension the fleet from a strategic point of view. Each customer $i \in N_{C}$ has pickup $\left(p_{i}\right)$ and delivery $\left(d_{i}\right)$ demands, with $0 \prec d_{i}, p_{i} \leq C V$. The problem is to determine the locations of depots, the assignment of customers to the opened depots and the corresponding vehicle routes with a minimum total cost under the following constraints:

(1) Each vehicle is used at most one route

(2) Each customer is served by exactly one vehicle

(3) Each route begins and ends at the same depot

(4) The total vehicle load at any point of the route does not exceed the vehicle capacity

(5) The total pickup and total delivery load of the customers assigned to an opened depot do not exceed the capacity of the depot

In this paper, we regarded a variant of the LocationRouting Problem with consideration of green aspects, namely the green LRP with simultaneous pickup and delivery. This specific problem seeks to minimize total cost by simultaneously locating the distribution centers and designing the vehicle routes that satisfy pickup and delivery demand of each customer at the same time, in a way that ecological aspects are observed. There are miscellaneous green criterion in literature. Our effort was to consideration of minimizing the weight of vehicle as a green criterion in order to minimize the effect of greenhouse gases. Besides, vehicles are heterogeneous in this paper. This assumption is exactly opposite of the research of Karaoglan et al (vehicles were homogeneous) [4].

\section{Mathematical Model}

The following notations are used for mathematical formulation of proposed model: 
$t m_{v \bar{c} c}^{c u s} \quad$ Time interval between customer $c$ and customer $\hat{c}$ passed by vehicle $v$

$d i s_{d c} \quad$ Distance between distribution center $d$ and customer $c$

$t m_{v d c} \quad$ Time interval between customer $c$ and distribution center $d$ passed by vehicle $v$

$\cos t_{d}^{\text {dist }} \quad$ Construction cost of distribution center $d$

$\cos t_{v}^{v e h} \quad$ Supply cost of vehicle $v$

$\mathrm{del}_{c} \quad$ Delivery demand of customer $c$

pic $_{c} \quad$ Pickup demand of customer $c$

$f_{v} \quad$ Amount of usage of fuel in return for distance unit of vehicle $v$

$\hat{f}_{v} \quad$ Amount of usage of fuel in return for transported load unit of vehicle $v$

$c^{\text {fuel } \quad \text { Cost of usage of fuel }}$

$M \quad$ A big number

Variables :

$x_{d}^{\text {dist }}$

$x_{v}^{v e h}$

Binary variable which is equal to 1 if distribution center $d$ is constructed

$\lambda_{v \widehat{c} c}$

Binary variable which is equal to 1 if vehicle $v$ is supplied

$\beta_{v d}$

Binary variable which is equal to 1 if vehicle $v$ firstly goes to customer $\hat{c}$ then goes to customer $c$

$a t_{v c}$

Binary variable which is equal to 1 if vehicle $v$ is assigned to distribution center $d$

$a t_{v c} \quad$ Entering time of vehicle $v$ to the location of customer $c$

$U_{v c} \quad$ Available amount of delivery load of vehicle $v$ before serving to customer $c$

$W_{v c} \quad$ Available amount of pickup load of vehicle $v$ after serving to customer $c$

mass $_{v c} \quad$ Available amount of load of vehicle $v$ before entering to the location of customer $c$

The mathematical formulation of proposed model is as follows:

$$
\begin{aligned}
& \text { Min } z^{\cos t}=c^{f u e l} \times \sum_{v, d, c>1}\left(f_{v}+\widehat{f}_{v} \times d e l_{c}\right) \times d i s_{d c} \times \lambda_{v 1 c} \times \beta_{v d} \\
& +c^{f u e l} \times \sum_{v, \hat{c}>1, c>1}\left(f_{v}+\widehat{f}_{v} \times \text { mass }_{v c}\right) \times d i s_{\hat{c} c}^{c u s} \times \lambda_{v \widehat{c} c} \\
& +c^{f u e l} \times \sum_{v, d, c>1}\left(f_{v}+\hat{f}_{v} \times p i c_{c}\right) \times d i s_{d c} \times \lambda_{v c 1} \times \beta_{v d} \\
& +\sum_{v} x_{v}^{v e h} \times \cos t_{v}^{v e h}+\sum_{d} x_{d}^{\text {dist }} \times \cos t_{d}^{d i s t}
\end{aligned}
$$

s. t:

$$
\begin{array}{lc}
\sum_{c} \text { pic }_{c} \leq \operatorname{cap}_{d}^{\text {dist }} \times x_{d}^{\text {dist }} & \forall d \\
\sum_{c} d e l_{c} \leq c a p_{d}^{\text {dist }} \times x_{d}^{d i s t} & \forall d \\
\operatorname{mass}_{v c} \leq c a p_{v}^{v e h} \times x_{v}^{v e h} & \forall v, c \\
\sum_{c} \operatorname{mass}_{v c} \leq M \times \sum_{d} \beta_{v d} & \forall v, d \\
\sum_{d} \beta_{v d} \leq 1 & \\
\sum_{v} \beta_{v d} \leq M \times x_{d}^{d i s t} & \forall d
\end{array}
$$




$$
\begin{aligned}
& \sum_{\hat{c}} \lambda_{v \bar{c} c} \leq 1 \\
& \sum_{\hat{c}} y_{v \widehat{c} c}=\sum_{\hat{c}} y_{v c \hat{c}} \quad \forall v, c \\
& a t_{v c} \geq \sum_{\bar{c}}\left(a t_{v \widehat{c}}+t m_{v \bar{c} c}^{c u s}\right) \times \lambda_{v \widehat{c} c} \quad \forall v, c>1 \\
& a t_{v c} \geq \sum_{d} t m_{v d c} \times \beta_{v d} \times \lambda_{v c 1} \quad \forall v, c \\
& \operatorname{mass}_{v c} \leq M \times \sum_{\hat{c}} \lambda_{v \widehat{c} c} \quad \forall v, c \\
& U_{v c}-U_{v \widehat{c}}+c a p_{v}^{v e h} \times \lambda_{v c \hat{c}}+\left(c a p_{v}^{v e h}-d e l_{\widehat{c}}-d e l_{c}\right) \times \lambda_{v c \hat{c}} \leq c a p_{v}^{v e h}-d e l_{\widehat{c}} \quad \forall v, c, \widehat{c} \\
& W_{v \widehat{c}}-W_{v c}+c a p_{v}^{v e h} \times \lambda_{v c \widehat{c}}+\left(c a p_{v}^{v e h}-p^{2} c_{\widehat{c}}-p i c_{c}\right) \times \lambda_{v c \widehat{c}} \leq c a p_{v}^{v e h}-p i c_{c} \quad \forall v, c, \widehat{c} \\
& U_{v c}+W_{v c}-d e l_{c} \leq c a p_{v e h}^{v} \quad \forall v, c \\
& U_{v \widehat{c}} \geq d e l_{\widehat{c}}+\sum_{c} d e l_{c} \times \lambda_{v \widehat{c} c} \quad \forall v, \widehat{c} \\
& W_{v c} \geq p i c_{c}+\sum_{\hat{c}} p i c_{\widehat{c}} \times \lambda_{v \widehat{c} c} \quad \forall v, c \\
& \operatorname{mass}_{v c}=\sum_{\hat{c}}\left(U_{v c}+W_{v \widehat{c}}\right) \times \lambda_{v \widehat{c} c} \quad \forall v, c \\
& x_{d}^{\text {dist }}, x_{v}^{v e h}, \lambda_{v \bar{c} c}, \beta_{v d} \in(0,1) \quad \forall d, v, c, \hat{c}, \\
& a t_{v c}, U_{v c}, W_{v c}, \text { mass }_{v c} \geq 0 \quad \forall v, c .
\end{aligned}
$$

Objective function minimizes transportation costs (usage of fuel and supply of vehicle) as well as the cost of construction of distribution centers. Constraints (1-2) guarantee that the total amount of pickup and delivery demand to customers by each distribution center in each period do not exceed the capacity of distribution center. Constraint (3) expresses that the amount of transported products by each vehicle do not exceed the capacity of vehicle. Constraint (4) explains that each vehicle is assigned to one distribution center. Based on constraint (5) each vehicle is at most assigned to one distribution center. Constraint (6) expresses that it is not possible to assign a vehicle to one distribution center, unless that distribution center is constructed. Constraint (7) assures that each vehicle visits each customer at most one in each period but it is possible to visit some vehicle simultaneously. Constraint (8) represents that if a customer enters to a specific location, he must leave there. Sub tour elimination is explained by constraints (9-10). Condition of receiving a product by customer is the visit of customer by vehicle which is expressed by constraint (11). Constraints (12-13) guarantee that delivery and pickup demand of customer are satisfied. Constraint (14) delineates that total transported load by each vehicle does not exceed the capacity of vehicle. Constraints (15-16) calculate the available amount of pickup and delivery demand of each customer. Constraint (17) shows the available amount of vehicle load before entering to the location of customer. Constraint (18-19) represents the range of model variables.

\section{Methodology and Numerical Experiments}

In this section, we evaluate the tractability of the proposed programming model in terms of the solution quality and the required computation time. To do this, we perform some numerical experiments on a set of randomly generated problem instances in small sizes. The programming models are implemented in GAMS 23.6 modeling language. All experiments are implemented on a Laptop with a Core 5 Duo CPU processor and 4 GB of RAM. The required input data of the test problems are presented in Table 1. For each test problem, the value of objective function and CPU time is reported in Table 2. In this table, when the number of indices increased, the objective value also increased. Although the 
computational time does not have a large amount in this problem, it is obvious that an efficient heuristic method should be used to obtain near-optimal solutions for more complicated problems.

Table 1. Some important inputs for models.

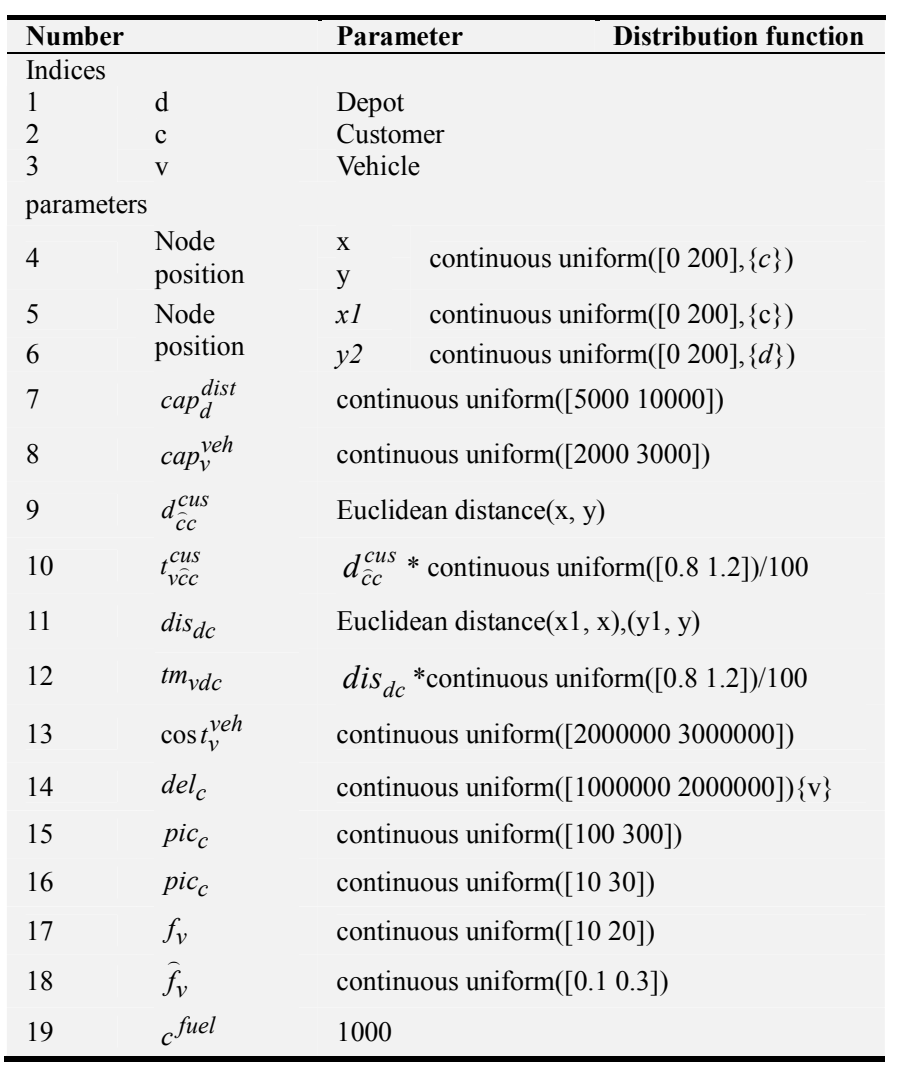

Table 2. Numerical results for a set of 7 test problems.

\begin{tabular}{llllll}
\hline \multirow{2}{*}{$\begin{array}{l}\text { Problem } \\
\text { number }\end{array}$} & \multicolumn{2}{l}{ Indices } & \multicolumn{3}{c}{ GAMS software } \\
\cline { 2 - 6 } & d & c & v & Objective value & CPU time \\
\hline 1 & 3 & 5 & 3 & 109195198 & 21.8 \\
2 & 3 & 6 & 3 & 121659765 & 32 \\
3 & 4 & 6 & 4 & 123146583 & 54.2 \\
4 & 4 & 7 & 5 & 143613035 & 138.8 \\
5 & 5 & 8 & 5 & 148069078 & 274.4 \\
6 & 6 & 8 & 5 & 150672113 & 485.7 \\
7 & 6 & 9 & 6 & 161538009 & 641.5 \\
\hline
\end{tabular}

\section{Conclusion}

In this paper, we regarded a variant of the LocationRouting Problem with consideration of green aspects, namely the green LRP with simultaneous pickup and delivery. This specific problem seeks to minimize total cost by simultaneously locating the distribution centers and designing the vehicle routes that satisfy pickup and delivery demand of each customer at the same time, in a way that ecological aspects are observed. The problem was formulated as a mixed integer programming model, which could then be solved using GAMS optimization software to determine the best vehicle routs and the optimal number of utilized vehicles. We provided a set of 7 test problems. For each test problem, the value of objective function and CPU time were reported. Although the computational time did not have a large amount in this problem, it is obvious that an efficient heuristic method should be used to obtain near-optimal solutions for more complicated problems.

\section{References}

[1] Christie, J S., Satir, S., \& Campus, T P (2006) Saving our energy sources and meeting Kyoto emission reduction targets while minimizing costs with application of vehicle logistics optimization In Proceedings of the Annual Conference of the Transportation Association of Canada Charlottetown, Prince Edward Island.

[2] Figliozzi, M A (2009) Planning approximations to the average length of vehicle routing problems with time window constraints Transportation Research Part B: Methodological, 43(4), 438-447.

[3] Ilgin, M A., \& Gupta, S M (2010) Environmentally conscious manufacturing and product recovery (ECMPRO): a review of the state of the art Journal of environmental management, 91(3), 563-591.

[4] Karaoglan, I., Altiparmak, F., Kara, I., \& Dengiz, B (2012) The location-routing problem with simultaneous pickup and delivery: Formulations and a heuristic approach Omega, 41(4), 465-477.

[5] Kuo, Y., \& Wang, C C (2011) Optimizing the VRP by minimizing fuel consumption Management of Environmental Quality: An International Journal, 22(4), 441 -451.

[6] Melo, M T., Nickel, S., \& Saldanha-Da-Gama, F (2009) Facility location and supply chain management- A review European journal of operational research, 196(2), 411 -412.

[7] Min, H (1989) The multiple vehicle routing problem with simultaneous delivery and pick-up points Transportation Research Part A: General, 23(5), 377-386.

[8] Pradenas, L., Oportus, B., \& Parada, V (2013) Mitigation of greenhouse gas emissions in vehicle routing problems with backhauling Expert Systems with Applications, 41(8), 2985-2991.

[9] Salhi, S., \& Sari, M (1997) A multi-level composite heuristic for the multi-depot vehicle fleet mix problem European Journal of Operational Research, 113(1), 95-112.

[10] Smith, H K., Laporte, G., \& Harper, P R (2009) Locational analysis: highlights of growth to maturity Journal of the Operational Research Society, s141 -s148.

[11] Subramanian, A., Drummond, L M D A., Bentes, C., Ochi, L S., \& Farias, R (2010) A parallel heuristic for the vehicle routing problem with simultaneous pickup and delivery Computers \& Operations Research, 37(11), 1899-1911.

[12] Tasan, A S., \& Gen, M (2012) A genetic algorithm based approach to vehicle routing problem with simultaneous pickup and deliveries Computers \& Industrial Engineering, 62(3), 755-761.

[13] Urquhart, N., Hart, E., \& Scott, C (2010) Building low CO 2 solutions to the vehicle routing problem with Time Windows using an evolutionary algorithm In Evolutionary Computation (CEC), 2111 IEEE Congress on (pp 1 -6) IEEE. 
[14] Xiao, Y., Zhao, Q., Kaku, I., \& Xu, Y (2012) Development of a fuel consumption optimization model for the capacitated vehicle routing problem. Computers \& Operations Research, 39(7), 1419-1431.

[15] Yong, P., \& Xiaofeng, W (2009) Research on a vehicle routing schedule to reduce fuel consumption In Measuring
Technology and Mechatronics Automation, 2119 ICMTMA'19 International Conference on (Vol 3, pp 825-827) IEEE.

[16] Zachariadis, E E., Tarantilis, C D., \& Kiranoudis, C T (2009) A guided tabu search for the vehicle routing problem with two-dimensional loading constraints European Journal of Operational Research, 195(3), 729-743. 